\title{
Avoiding BAK in postoperative eye drops reduces the need for subconjunctival 5-FU injections post-trabeculectomy
}

\author{
Keith Ong ${ }^{1,2,3,4}$, Leonard Ong ${ }^{4,5,6}$ \\ 'Department of Ophthalmology, Northern Clinical School, University of Sydney, \\ Sydney, Australia; '2 Department of Ophthalmology, Royal North Shore Hospital, \\ Sydney, Australia; ${ }^{3}$ Department of Ophthalmology, Sydney Adventist Hospital, \\ Sydney, Australia; ${ }^{4}$ Department of Ophthalmology, Chatswood Private Hospital, \\ Sydney Australia; ${ }^{5}$ Ophthalmology Clinic, Macquarie University Hospital, Sydney, \\ Australia; ${ }^{6}$ Department of Clinical Medicine, Faculty of Medicine and Health \\ Sciences, Macquarie University, Sydney, Australia
}

\begin{abstract}
Purpose: Subconjunctival fibrosis is one of the main causes of failure of glaucoma filtration surgery. It can result in absence of a filtration bleb, a small scarred bleb, or a cystic bleb. 5-Fluorouracil (5-FU), mitomycin C (MMC), and topical steroids have been used to suppress subconjunctival fibrosis.

Method: A study was done analyzing the number of postoperative subconjunctival 5-FU injections for trabeculectomy on pseudophakic eyes prior to and following the change to a BAK-free regimen. The cohort consisted of 16 consecutive cases undergoing primary trabeculectomy without intraoperative MMC or 5-FU. The trabeculectomy surgery included a groove sclerectomy procedure.

Group A were 8 eyes of patients who had the author's standard Chlorsig, Maxidex, and Prednefrin Forte eye drops tds. Group B were 8 eyes who had Chlorsig-dexamethasone and Optive-dexamethasone tds eye drops postoperatively.

Results: Group B (BAK-free) patients required fewer postoperative 5-FU subconjunctival injections (average: 2.9, range: 1-5 injections) compared to Group A (BAK) patients (average: 7.3, range: 4-18 injections). This difference was statistically significant $(P=0.02$, unpaired t-test).

All patients had functioning blebs and did not require glaucoma medications to maintain target intraocular pressure. The Group B (BAK-free) patients had more diffuse blebs than the Group A (BAK) patients.
\end{abstract}

Conclusion: The results demonstrated that when BAK was eliminated from postoperative eye drops in trabeculectomy, the number of postoperative 5-FU injections was reduced.

Correspondence: Dr. Keith Ong, 2 Railway Avenue, Eastwood NSW 2122, Sydney, Australia.

E-mail: keithong@optusnet.com.au 


\section{Introduction}

Subconjunctival fibrosis is one of the main causes of failure in glaucoma filtration surgery. It can result in absence of a filtration bleb, a small scarred bleb, or a cystic bleb. 5-Fluorouracil (5-FU), mitomycin C (MMC) and topical steroids have been used to suppress subconjunctival fibrosis.

In patients with meibomian gland dysfunction, or inflammation related to long -term use of glaucoma eye drops containing benzalkonium chloride (BAK), subconjunctival fibrosis is more prevalent. ${ }^{1}$

\section{Background}

The author had a trabeculectomy patient who was allergic to BAK. All the commonly used postoperative steroid eye drops such as Maxidex (Alcon), Prednefrin Forte (Allergan), FML (Allergan), Flucon (Alcon), Flarex (Alcon) contain BAK, and hence, could not be used. Siguent Hycor 1\% (Aspen Pharma) eye ointment was used as it did not contain BAK preservative. As this would not give sufficient anti-inflammatory effect, two vials of dexamethasone for injection (Mylan) (1 $\mathrm{ml}$ of 4 $\mathrm{mg} / \mathrm{ml}$ dexamethasone) was added to Chlorsig (Aspen Pharma) eye drops (10 $\mathrm{ml}$ ) and also Optive eye drops (15 ml). Chlorsig contain phenyl mercuric acetate preservative and Optive (Allergan) contain purite preservative. Both of these dexamethasone BAK-free combination drops were used postoperatively $t d s$. As this regimen resulted in a favorable functioning bleb requiring only five postoperative 5 -FU injections, it was decided to use this BAK-free regimen for all patients from September 2018.

Mild BAK allergy and toxicity usually does not cause much conjunctival inflammation because the steroid eye drops mask the inflammatory reaction.

\section{Method}

A retrospective audit study was done analyzing the number of postoperative subconjunctival 5-FU injections for trabeculectomy on pseudophakic eyes prior to and following the change to a BAK-free regimen. The consecutive cases analyzed were for primary trabeculectomy on pseudophakic eyes and did not have intraoperative MMC or 5-FU. The trabeculectomy surgery included a groove sclerectomy procedure. ${ }^{2}$ Patients having combined cataract phacoemulsification and trabeculectomy and revision trabeculectomy were excluded.

\section{Results}

The cohort (Table 1) consisted of 16 consecutive eyes which had trabeculectomy, were pseudophakic, and of Chinese ethnicity. Group A comprised 8 eyes of patients with average age 70.4 years with range of 64 to 83 years of age who 
Table 1. Group comparison of postoperative 5-FU injections and age

\begin{tabular}{|l|l|l|l|l|}
\hline & \multicolumn{2}{|l|}{ Group A (with BAK) } & \multicolumn{2}{l|}{ Group B (BAK-free) } \\
\hline & $\begin{array}{l}\text { Postoperative } \\
\text { 5-FU }\end{array}$ & Age & $\begin{array}{l}\text { Postoperative } \\
\text { 5-FU }\end{array}$ & Age \\
\hline Patient 1 & 7 & 83 & 5 & 68 \\
\hline Patient 2 & 18 & 65 & 3 & 93 \\
\hline Patient 3 & 4 & 66 & 2 & 66 \\
\hline Patient 4 & 7 & 67 & 1 & 81 \\
\hline Patient 5 & 4 & 71 & 4 & 64 \\
\hline Patient 6 & 6 & 80 & 1 & 64 \\
\hline Patient 7 & 5 & 67 & 5 & 66 \\
\hline Patient 8 & 7 & 64 & 2 & 79 \\
\hline
\end{tabular}

had the author's standard Chlorsig, Maxidex, and Prednefrin Forte eye drops tds. Group B comprised 8 eyes of patients with average age 72.6 years and range from 64 to 93 years of age who had Chlorsig-dexamethasone and Optive-dexamethasone tds eye drops postoperatively.

\section{Discussion}

Group A and Group B did not differ significantly in age ( $P=0.62$, unpaired t-test).

Group B (BAK-free) patients required fewer postoperative 5-FU subconjunctival injections (average: 2.9, range: 1-5 injections) compared to Group A (BAK) patients (average: 7.3, range: 4-18 injections). This difference was statistically significant $(P=0.02$, unpaired t-test). If the patient with the highest number of 5-FU injections is excluded from each group, since patient 2 in Group A had a much higher number compared to all patients and may have led to this statistical significance, an even more statistically significant result is obtained (2.6 in BAK-free versus 5.7 in BAK groups, $\mathrm{P}=0.002$ ).

All patients had functioning blebs and did not require glaucoma medications to maintain target intraocular pressure. The Group B (BAK-free) patients had a more diffuse bleb than the Group A (BAK) patients.

The results demonstrated that when BAK was eliminated from postoperative eye drops in trabeculectomy, the number of postoperative 5FU injections was reduced. 


\section{Conclusion}

Changing the postoperative eye drops to exclude BAK does not pose any risk and could increase the success of all glaucoma surgery with bleb formation. It would improve the outcome of minimally invasive glaucoma surgical procedures such as the Xen implant by reducing the number of postoperative bleb needling and injections. Furthermore, avoiding BAK in glaucoma drops that have to be used following bleb surgery could increase the long-term survival of blebs.

In conclusion, this study is consistent with reduced subconjunctival fibrosis when BAK is avoided postoperatively. A practical method of preparing steroid eye drops without BAK has been described and would be useful until BAK-free steroid eye drops are easily available and affordable.

\section{References}

1. Steven DW, Alaghband P, Lim KS. Preservative in glaucoma medication. Br J Ophthalmol 2018;102:1497-1503.

2. Ong K. Groove sclerectomy in trabeculectomy. YouTube video;2018. Available at: https://www. youtube.com/watch?v=uFtW4aozNTY 